\title{
Spandex Shoji Synthesizer Transforming Elastic Interaction into Images and Sounds
}

\author{
Shumpei Tamura, Yasuo Kuhara* \\ Department of Interactive Media, Tokyo Polytechnic University, Japan \\ *kuha@int.t-kougei.ac.jp
}

\begin{abstract}
We developed an interactive artwork generating images and sounds using the traditional Japanese interior fixture known as Shoji. We used spandex fiber, which is a polyurethane fiber having remarkable elasticity, as a Shoji screen. When we push and expand the elastic fiber using our hand, it generates images and sounds according to the degree of its expansion and contraction. The images are projected onto the area of the Shoji as a screen touched by a performer. We can enjoy the interaction of the elastic feeling synchronized with the images and sounds by touching the Shoji screen.
\end{abstract}

Keywords: interactive art, sound Installation, shoji, spandex, elasticity, Kinect

\section{Introduction}

Shoji is a traditional Japanese interior fixture. It is a sliding door or window made of wooden lattice pasted with Japanese paper to make a screen. In contrast to a normal door, the translucency of its screen enables more light to be let in while it is closed. Additionally, different from a window, because of its lack of transparency we cannot clearly see objects on the other side of the screen but only their silhouette images depending on the light condition. Because of these characteristics, Shoji is a typical interior fixture directing the Japanese beauty of shade and shadow in a dim light.

The Japanese have a proverb: "Walls have ears; Shoji have eyes." This saying represents a peeping person, who rips the paper to make a small hole in the Shoji by poking it with their finger in order to look at the other side through it. Adequate translucency of Shoji stimulates a feeling of curiosity about the other side.

Some projection mapping works have used Shoji as a screen, for example, "Shouji ni Mary." However, these are movies that are mainly for watching without touching the Shoji. On the other hand, "Fire wall" is an interactive artwork whereby we can touch the screen made of soft fabric causing projection of images onto the screen and playing of music. In this work, the action of pushing the fabric is used to trigger the playing of movies and change the speed and volume of music based on the depth, therefore the degree of pushing strength is not reflected in musical expressions of performers. Additionally, "Humanaquari$u^{\prime \prime}$ "is a movable performance space with solid touch screens combined with projection responding to audience interaction. In this work, players can not feel a soft and elastic touch because of a glass window.

In the case of Shoji, when you push the paper of the screen with your finger, you can feel the elasticity of the Shoji against your finger because of its stretching properties. The harder you push it, the greater the feeling of tension against your finger whether the paper is ripped or not. Our work was inspired by Shoji filled with a Japanese emotional atmosphere, and we pursue the graphical and musical ex- 
pression of the sense of tension arising from the elasticity when we touch Shoji.

\section{Concept}

This work is an interactive artwork that reacts to poking the Shoji screen with one's finger. We emphasize this action of "poking Shoji with one's finger." If you have ever seen Shoji in childhood, you have probably felt the temptation to rip the Shoji paper by poking it with your finger. This feeling may arise from certain causes, which are curiosity about the other side of the screen and the material of soft paper in which a hole can be easily made. Shoji as an interior fixture has many factors stimulating various emotions. This work aims to express these deep feelings for Shoji.

This work uses spandex fiber, which is made of strongly elastic fabric, for the Shoji screen. In the case of paper, we have to repair it each time someone rips the paper of the screen. Using white spandex fiber, we can enjoy the action of poking Shoji with our finger repeatedly while keeping the appearance of the Shoji. Additionally, we can enjoy the elastic force of this fabric, and we use it as a method of interactive art transforming the elasticity into images and sounds.

\section{Configuration}

This work comprises projection mapping onto a spandex fiber screen attached to the wooden lattice of Shoji, which is configured by a grid square with nine cells of $3 \times 3$ as shown in Figure 1. Spandex is made from polyurethane fiber known for its extraordinary elasticity. Because of this feature, it is often used for sportswear. This work is composed of a Shoji main unit, Microsoft Kinect for a depth sensor measuring the displacement of the screen, a projector, speakers, and a PC controlling the entire system as shown in Figure 2. We use Max/MSP/Jitter software and jit.freenect.grab of its external object for grabbing the Kinect image.

When you push the Shoji screen with your finger, images and sounds synchronized with the elasticity that you feel are made. The

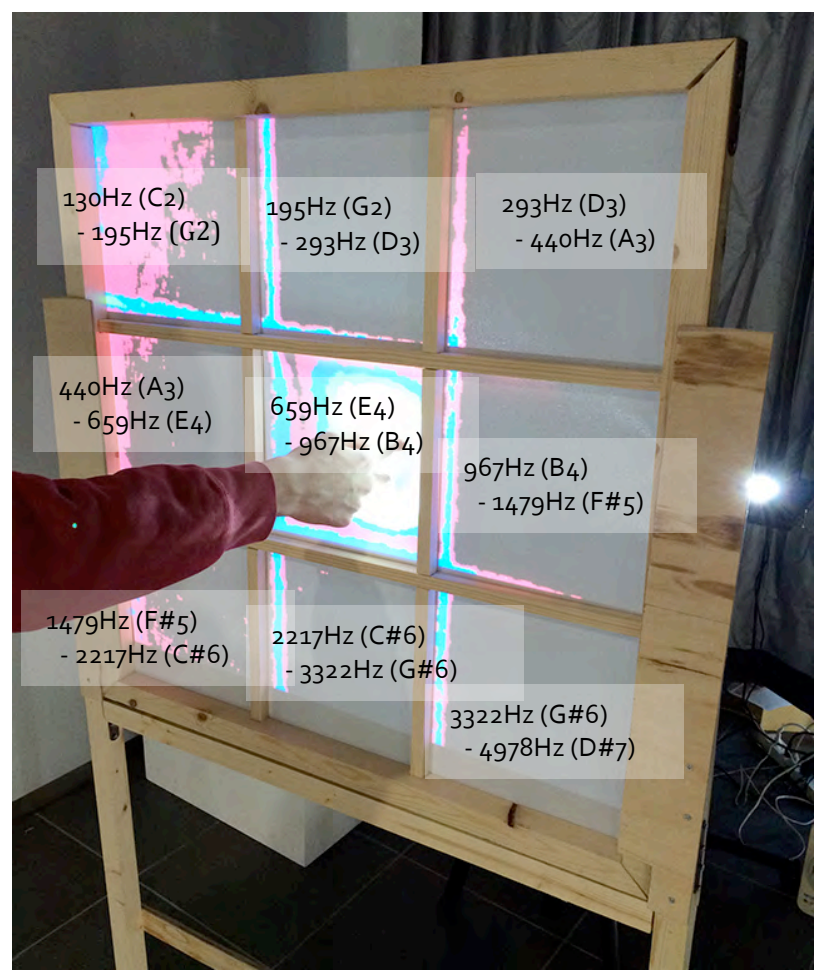

Figure 1. Overview and example of frequency bands.

depth sensor of Kinect, which is located on the other side, measures the deviation from the normal position of the screen in proportion to how hard you push it with your finger. The deviation is transformed to images and sounds. As images, contours of the color gradient corresponding to the deviation are drawn on the screen as shown in Figure 3. For example, if you push the screen hard, the color of the image is displaced toward yellow, and if you push the screen gently, the color of the image is displaced toward red.

At the same time, the sound of sine waves is generated with images as described above. Nine different frequency bands are allocated to the nine square grid cells of the Shoji screen as shown in Figure 1. For example, we assigned various perfect fifth intervals, which are 130 to $195 \mathrm{~Hz}, 195$ to $293 \mathrm{~Hz}, 293$ to $440 \mathrm{~Hz}, 440$ to $659 \mathrm{~Hz}, 659$ to $967 \mathrm{~Hz}$, etc. If you push a square cell of the Shoji, the sound of the sine wave with the allocated frequency are generated. If you push a cell deeply, the pitch of the sound is shifted higher in proportion to the deviation of the cell made by your hand. If you push multiple cells, a sound synthesized with multiple sine waves of different frequencies is made. You can feel the synchronization of the 


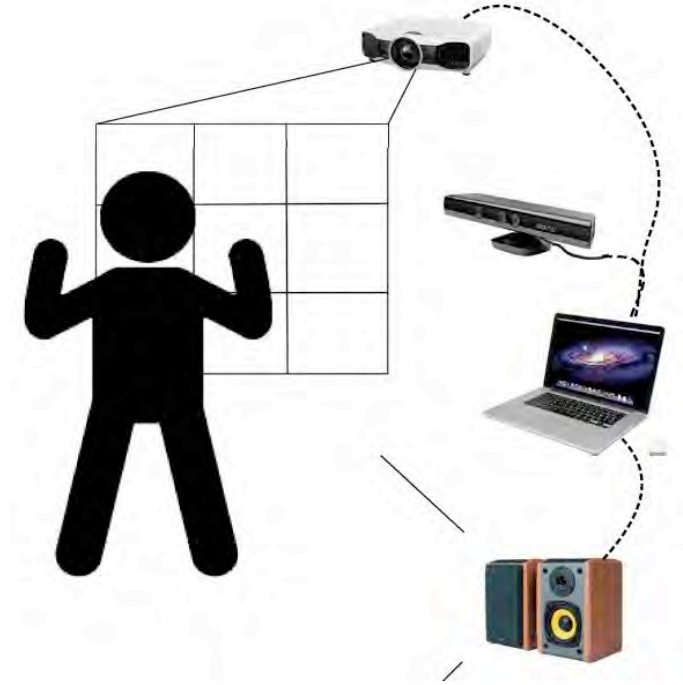

Figure 2. System configuration.

deviation of the screen that you push and the sound made, because the pitch of the sound corresponds to the force with which you push.

In this way, we can enjoy various images and sounds synchronized with the elasticity by touching the Shoji.

\section{Performance}

First, a performer stands in front of the Shoji and pushes the Shoji screen made of spandex. Then, the Kinect sensor detects the deviation of the screen, which is transformed into certain values for generating images and sounds. Next, the sound of the sine wave with the frequency according to the deviation is made. At the same time, contour images of the color gradient corresponding to the deviation are projected onto the cell of the screen touched by the performer.

Because of the extreme elasticity of spandex fiber, the screen expands and contracts in sensitive response to the performer's action of pushing or releasing. The surface of the screen wrinkles or fluctuates as you push hard or more gently. This fluctuation creates graphical effects such as ripples sensitively reflecting the degree of force by pushing the screen.

Moreover, you can poke the screen in the same way as ripping it to make a small hole in the paper of the Shoji with your finger. Paper is damaged if you push it too hard. However, in the case of spandex fiber, the screen is not damaged and can expand it to a large extent even if you push pretty hard. The harder you push, the more you feel the action-reaction force and the higher the pitch of the sound that is gradually shifted. As a result, this work generates a unique sense of tension similar to ripping Shoji paper with your finger. You can watch some movies of our works on Internet, at URL http://www.kuhalabo.net/shojisynth/.

\section{Conclusion}

We developed an interactive artwork expressing the features of Shoji, which is a traditional Japanese interior fixture. We enjoy the feeling of tension in elasticity caused by pushing the screen as well as the images and sounds synchronized with our action. In future, we will improve the color gradient to be finer by making the resolution higher. Additionally, we will install a low-frequency oscillator, or LFO, to some square cells of the screen, and generate more expressive sounds by synthesizing various waves.
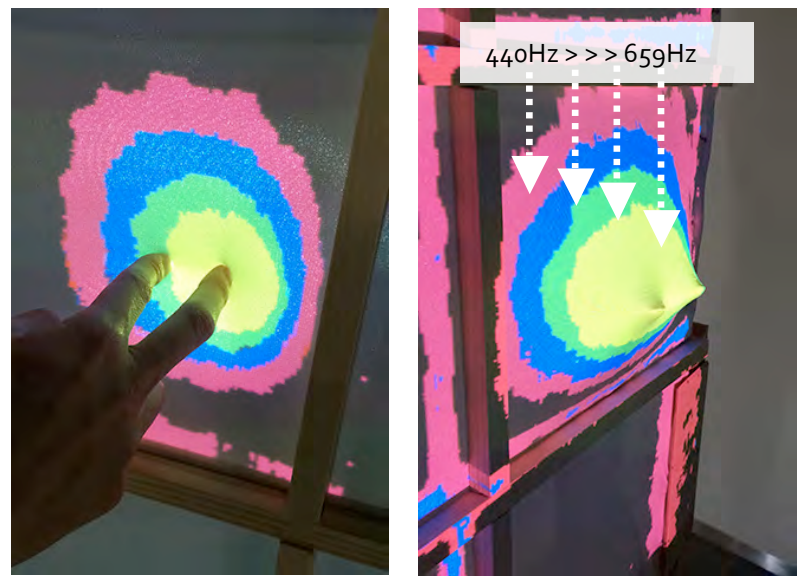

Figure 3. Image mapping and example of pitch shift.

\section{References}

Noya Kudo (2012). "Shouji ni Mary". URL http://yeah7o.tumblr.com/

Aaron Schwarz (2013). "Firewall". Future Interfaces Conference, New York 2013.

Robyn Taylor et al. (2011). "Designing from within: humanaquarium", The ACM Conference on Human Factors in Computing Systems 2011. 